\title{
Análise de atividades e formação do terapeuta ocupacional: um estudo com os preceptores de estágio da UFPE*
}

\author{
Activity analysis and education of occupational \\ therapists: a study with intern preceptors at the \\ Federal University of Pernambuco, Brazil
}

\author{
Daniela Sá Leitão Guimarães ${ }^{1}$, Ilka Veras Falcão²
}

\begin{abstract}
GUIMARÃES, D. S. L.; FALCÃO, I. V. Análise de atividades e formação do terapeuta ocupacional: um estudo com os preceptores de estágio da UFPE. Rev. Ter. Ocup. Univ. São Paulo, v. 15, n. 2, p. 63-70, maio/ago., 2004.

RESUMO: No Brasil os primeiros cursos de graduação de Terapia Ocupacional foram criados no final dos anos 50 e início dos anos 60. Em 1963, constituiu-se, por aprovação do Conselho Nacional de Educação, o Currículo Mínimo dos cursos de Terapia Ocupacional e, em 1982, esse currículo foi modificado, fortalecendo a especificidade da profissão. No início dos anos 2000 , ocorre nova mudança, com a aprovação das Diretrizes Curriculares, que indica, entre outros conteúdos, a necessidade do estudo acerca das atividades humanas e a análise dessas atividades. O objetivo desta pesquisa é identificar o percurso desde a formação profissional à aplicação da análise de atividade na prática do terapeuta ocupacional preceptor de estágio da UFPE. É um estudo descritivo de campo, realizado com 28 preceptores de estágio curricular do curso de Terapia Ocupacional da Universidade Federal de Pernambuco. Dos pesquisados, $96 \%$ são do sexo feminino, com tempo de atuação de 10 a 19 anos (64\%), com predomínio nas áreas de Saúde Mental e Neurologia. A maioria (88\%) dos entrevistados refere ter estudado análise de atividade durante a graduação Todos os entrevistados afirmam ser necessário o estudo da análise de atividade para o terapeuta ocupacional e $84 \%$ realizam a análise em seu cotidiano profissional. Fica evidente que a análise de atividades, além de um conhecimento encontrado na literatura, na legislação profissional e na formação do terapeuta ocupacional, está também presente na prática cotidiana do profissional preceptor de estágio da UFPE.
\end{abstract}

DESCRITORES: Terapia ocupacional/educação. Terapia ocupacional/recursos humanos. Currículo/tendências.

\footnotetext{
* Este artigo é parte integrante do trabalho de conclusão de curso da Graduação em Terapia Ocupacional na Universidade Federal de Pernambuco. Novembro, 2003.

1 Terapeuta Ocupacional da AACD de Pernambuco. Professora substituta do Departamento de Terapia Ocupacional da Universidade Federal de Pernambuco.

2 Professora assistente do Departamento de Terapia Ocupacional da Universidade Federal de Pernambuco, Mestre em Saúde Pública.

Endereço para correspondência: Daniela S. L. Guimarães, Av. Gov. Carlos de Lima Cavalcante, 2747 - Casa Caiada, Olinda, PE CEP: 53130530. e-mail: danielaguimaraes@terra.com.br
} 


\section{A FORMAÇÃO DOTERAPEUTA OCUPACIONAL ESUA ESPECIFICIDADE}

$\mathrm{A}$ formação de terapeutas ocupacionais no Brasil se inicia no final de 1950 e no começo de 1960, quando foram criados os primeiros cursos de graduação na Universidade de São Paulo e na Associação Brasileira Beneficente de Reabilitação, no Rio de Janeiro (SOARES, 1991; CASTRO et al., 2001). No Nordeste, a formação de terapeutas ocupacionais aconteceu, de forma pioneira, em Pernambuco, com a abertura do curso para técnicos em Terapia Ocupacional em 1962 (PESSOA, 1992).

Embora já houvesse registro do uso de ocupações em hospitais psiquiátricos os cursos recémcriados, especialmente influenciados pela experiência norte-americana, voltaram-se para a área física para atender à demanda crescente das entidades e serviços de reabilitação. Segundo Soares (1991), os terapeutas ocupacionais formados nas primeiras escolas eram fortemente ligados à reabilitação física e à direção médica $\mathrm{e} o$ atendimento a doentes mentais era restrito à teoria ou a casos associados à deficiência física.

Para Castro et al. (2001), a formação que foi inicialmente estabelecida no Brasil visava a um profissional com capacidade para definir objetivos e técnicas terapêuticas diferenciadas pela patologia do paciente e especialidade médica associada. Esta característica só foi sendo alterada quando os terapeutas ocupacionais se organizaram e, então, ao final do ano de 1963, constituiu-se o primeiro Currículo Mínimo do curso de Terapia Ocupacional aprovado pelo então Conselho Federal de Educação (hoje, Conselho Nacional de Educação) (FERRIGNO, 1991; PALHARES, 1991; DE CARLO; BARTALOTTI, 2001).

Outros fatos contribuíram para a formação do terapeuta ocupacional, como o reconhecimento da profissão como sendo de nível superior (Decreto-lei n ${ }^{\circ}$ 938/1969) e a criação, em 1975, do sistema de Conselho Federal e Conselhos Regionais, que, além da função de fiscalizar e normatizar as profissões de fisioterapeuta e terapeuta ocupacional, definiu suas competências e passou a influir na formação profissional. As discussões a esse respeito foram se ampliando e ganhando legitimidade no âmbito político, profissional e acadêmico com a participação de docentes, escolas e associações profissionais, entre outras instituições (GILL, 1982; FINGER, 1986; PALHARES, 1991).

Como resultado de um amplo debate nacional, em 1982, o Currículo Mínimo de Terapia Ocupacional foi modificado, tendo sido adotado um modelo, mais extenso, com carga horária que passou de 2160 para 3240 horas (PALHARES, 1991; PESSOA, 1992). Para Emmel (2001), este novo currículo fortaleceu a especificidade da profissão, a medida em que ampliou o número de disciplinas tanto na área profissionalizante, quanto na de fundamentos de Terapia Ocupacional. Ainda conforme a autora, os anos 1990 foram marcados pela capacitação formal (pós-graduação stricto-sensu) dos docentes terapeutas ocupacionais, o que também trouxe um incremento à formação. Uma importante conseqüência dessa capacitação e da melhoria da formação profissional foi o início e reconhecimento da produção científica em Terapia Ocupacional.

No final da década de 1990 e início dos anos 2000 , ocorreu nova mudança em relação às normas para a abertura de cursos superiores, com aprovação das Diretrizes Curriculares visando oferecer uma formação básica e preparar o futuro graduado para enfrentar os desafios das rápidas transformações da sociedade, do mercado de trabalho e das condições de exercício profissional (BRASIL, 1997). Em relação ao curso de Terapia Ocupacional, a Resolução N ${ }^{\circ}$ 06, do Conselho Nacional de Educação (BRASIL, 2002), estabelece como conteúdos essenciais os que envolvem as Ciências Biológicas e da Saúde, as Ciências Sociais e Humanas e as da Terapia Ocupacional.

Analisando os conteúdos específicos para a formação do terapeuta ocupacional nas Diretrizes Curriculares e também na literatura, verificamos que o que confere especificidade à formação é o conhecimento das atividades e a experiência de analisar essas atividades.

Segundo Crepeau (2002, p. 132):

o aprendizado da análise de atividade começa no início de educação em Terapia Ocupacional nos níveis voltados para tarefa e a teoria. O trabalho de campo e a prática profissional desenvolvem melhor e aperfeiçoam estas habilidades quando os profissionais a utilizam com seus clientes.

A mesma autora, acrescenta ainda, que a análise de atividade é realizada pelos terapeutas ocupacionais para a compreensão das atividades, de suas partes componentes e dos significados culturais a elas atribuídos. Afirma que a análise de atividade contribui para o raciocínio clínico dos profissionais de Terapia Ocupacional, porém diz: "A análise da atividade é um aspecto da prática clínica tão automático que freqüentemente é ignorado ou não observado" (p.121).

Ainda de acordo com Crepeau (2002), a análise de atividades ocorre em três níveis, com ênfase na tarefa, ênfase na teoria e ênfase no individuo. A ênfase na tarefa refere-se às habilidades necessárias para executar 
as atividades, observando-se seu significado cultural e potencial terapêutico, o que representa a forma como "os estudantes inicialmente aprendem a analisar a atividade" (p. 121). A análise de atividade voltada para a teoria também faz parte da formação, pois o estudante aprende teorias específicas de Terapia Ocupacional e como planejar o tratamento de acordo com essas teorias. Já a análise voltada para o individuo, não está centrada na atividade e sim, no cliente, levando em consideração os interesses, as capacidades, as limitações funcionais da pessoa e o contexto de desempenho temporal e ambiental. Portanto, é o tipo de análise realizada pelo profissional.

Portanto, o estudo e realização de análise de atividades são centrais para o terapeuta ocupacional. No entanto, nos questionamos sobre a forma como, em nosso contexto local, esse conhecimento específico está sendo assimilado, qual a sua contribuição para a formação profissional e como se dá a aplicação de tais conhecimentos na atuação do terapeuta ocupacional em Pernambuco.

Assim, perguntamos: será que os terapeutas ocupacionais aplicam o conhecimento de análise de atividades, ou o mesmo é importante para a formação (construção), porém não se aplica no cotidiano profissional?

Dessa forma, é objetivo deste estudo identificar o percurso desde a formação profissional à aplicação da análise de atividade na prática do terapeuta ocupacional preceptor de estágio da UFPE.

\section{CASUÍSTICA EMÉTODO}

Realizamos um estudo descritivo de campo com 28 terapeutas ocupacionais, preceptores de estágio curricular do Curso de Terapia Ocupacional da Universidade Federal de Pernambuco (UFPE), distribuídos na Região Metropolitana do Recife (RMR), conforme listagem da Coordenação de Estágio do Curso em dezembro de 2002.

A opção por essa clientela considerou o fato de que tais terapeutas ocupacionais participam da formação no momento intermediário entre a universidade e o campo profissional e também por serem profissionais inseridos no mercado de trabalho há, no mínimo, dois anos, o que permitiria, pela experiência acumulada, optar pela realização ou não da análise de atividades conforme aprendido.

Por envolver seres humanos, o projeto de pesquisa foi aprovado pelo Comitê de Ética em Pesquisa (CEP) do Centro de Ciências da Saúde (CCS), da Universidade Federal de Pernambuco (UFPE), e as condutas para a coleta de dados e para a apresentação dos resultados foram regidas pela Resolução no 196/96, do Conselho Nacional de Saúde (BRASIL, 1996).

A pesquisa foi realizada em um período de 6 meses com 25 entrevistados $(89,3 \%)$. Os profissionais responderam a um questionário auto-aplicável, com 24 questões abertas e/ou fechadas, divididas em três sessões: com identificação e informações gerais; área de atuação e de formação profissional, abrangendo a aprendizagem e a aplicação da análise de atividades como estudante e, agora, como profissional. Os entrevistados podiam assinalar mais de uma alternativa em algumas das questões, ultrapassando, assim, o percentual de $100 \%$ na totalização das respostas.

Os dados da pesquisa foram analisados de acordo com a distribuição da freqüência simples e correlacionados à literatura, não sendo possível o tratamento estatístico em razão do pequeno universo de pesquisa.

\section{RESULTADOS}

Nosso estudo aponta que $96 \%$ dos entrevistados são do sexo feminino. Quanto à idade, esta varia de 27 a 53 anos, com média de 38 anos. Em relação ao tempo de conclusão do curso e de atuação profissional na área, estes são similares, com um mínimo de 3 anos e o máximo de 30 anos, com tempo médio de formação e atuação de 12 anos.

Quanto à qualificação profissional, a maioria dos entrevistados afirma ter realizado algum curso após a graduação (72\%). Pouco mais de $70 \%$ realizaram cursos de curta duração (Extensão e Aperfeiçoamento), 50\% cursaram Especialização e 8\% o Mestrado.

No que diz respeito à área de atuação, constatamos o discreto predomínio de profissionais atuantes na área de Saúde Mental (44\%) e Neurologia $(40 \%)$, sendo freqüente o profissional atuar concomitantemente em mais de uma área.

Com relação à clientela assistida, obtivemos respostas que se sobrepõem, com atendimentos a adultos $(76 \%)$, idosos (68\%), adolescentes $(40 \%)$ e crianças $(36 \%)$. Quanto à modalidade de atendimento, também é possível o profissional participar de mais de uma modalidade assistencial, com a maioria dos profissionais ( $80 \%$ ) em ambulatório, $44 \%$ em enfermaria, $16 \%$ em atendimento domiciliar e $8 \%$ em serviços de emergência. Quanto à dinâmica de atendimento, constatamos que os profissionais, em sua maioria (64\%), usam tanto o atendimento individual como grupal, indistintamente.

Com relação às atividades mais utilizadas no atendimento terapêutico ocupacional, observa-se o predomínio das auto-expressivas $(72 \%)$, das atividades de 
vida diária e funcionais $(48 \%)$, das artesanais e cognitivas ou pedagógicas ( $40 \%$ cada).

No que se refere ao uso de um modelo teórico que oriente a prática clínica, verificamos que $72 \%$ relatam utilizá-lo, enquanto que $12 \%$ não utilizam e $16 \%$ não responderam. Em relação à compreensão que os entrevistados têm a respeito do que seja e do uso da análise de atividades $4 \%$ não responderam, enquanto $56 \%$ afirmam ser o meio para conhecer as características das atividades, os seus benefícios e contra-indicações; 20\% afirmam ser o mecanismo para indicar, selecionar e identificar o objetivo terapêutico das atividades e $20 \%$ reconhecem a análise de atividades como uma forma de relacionar o interesse e a necessidade do cliente com o objetivo terapêutico de desenvolvimento de habilidades e desempenho de funções.

Quanto ao conhecimento da análise de atividades na formação, observou-se que a maioria respondeu afirmativamente (88\%), $8 \%$ negativamente e $4 \%$ não responderam. Por outro lado, todos os entrevistados disseram ser este conhecimento necessário para a atuação como terapeuta ocupacional, sendo que $88 \%$ afirmaram ser ele muito importante (atribuindo valor em uma escala de importância entre 9 e 10) e 12\% relataram ser importante (atribuindo valor entre 7 e 8).

No que diz respeito à escolha das atividades para uso com sua clientela, a maioria (52\%) dos profissionais respondeu que selecionam as atividades terapêuticas a partir do interesse, da necessidade ou decisão grupal do(s) cliente(s), e $48 \%$ escolhem por meio dos objetivos terapêuticos, benefícios previstos e aptidões do paciente. Outros critérios para a seleção de atividades dizem respeito aos recursos financeiros e ambientais disponíveis.

Quanto à realização da análise de atividades 16\% dos entrevistados não o fazem, enquanto $84 \%$ ressaltam fazer a análise, seja rotineira ou eventualmente, embora a maioria $(76 \%)$ não use um roteiro específico. Entre os terapeutas ocupacionais que não realizam análise de atividades, $8 \%$ alegam ser desnecessária devido à sua experiência profissional e os demais expressam motivos diversos. Quanto às circunstâncias em que fazem a análise, verificou-se que $68 \%$ realizam antes e durante os atendimentos, $64 \%$ quando utilizam atividades novas, $60 \%$ antes da indicação da atividade para o paciente e $12 \%$ apontam outros motivos.

\section{DISCUSSÃO}

A amostra estudada constitui-se de terapeutas ocupacionais predominantemente do sexo feminino, em concordância com estudos realizados com essa categoria profissional por Garcia (1997), Rosa e Emmel (2001) e Souza (1997). Segundo De Carlo e Bartalotti (2001), tal característica está presente desde o início da profissão, quando as mulheres eram escolhidas para exercêla por que seus traços maternais seriam benéficos ao tratamento.

Quanto à idade, observou-se neste estudo a prevalência da faixa etária de adultos jovens, com média de idade de 38 anos, o que é semelhante ao trabalho de Souza (1997), que obteve 61,8\% nessa faixa etária. Na pesquisa de Rosa e Emmel (2001), com terapeutas ocupacionais docentes, a média de idade é de 39 anos, o que também é similar ao nosso achado.

Com relação ao tempo de conclusão do curso e de atuação profissional na área, encontramos similaridade nesses dois dados, com variação entre 3 e 30 anos. Quanto à atuação profissional, houve maior percentual (64\%), na faixa de 10 a 19 anos de atuação, com tempo médio de 12 anos. Souza (1997), em seu estudo, encontrou maioria $(74,5 \%)$ de profissionais com menos de 15 anos de formação, o que pode ser explicado pelo crescimento das escolas de Terapia Ocupacional nos anos 80 e 90 e entrada no mercado após este período (VITTA, 1998).

Em nosso caso, esse fato pode não ter relação com a ampliação do número de cursos, mas com o aumento da demanda e da redução da evasão observada no Curso de Terapia Ocupacional da $\operatorname{UFPE}^{(1)}$ a partir do início dos anos 1990, o que fez crescer o número de terapeutas ocupacionais no mercado e Região Metropolitana do Recife.

Constatamos que $72 \%$ dos entrevistados realizaram algum curso após a graduação evidenciando interesse em se qualificar e/ou atualizar, mesmo já estando com seu lugar assegurado no mercado de trabalho. O predomínio de cursos latu senso (atualização e especialização) pode ser justificado pelas opções restritas de cursos sticto senso (mestrado e doutorado) abertos ao terapeuta ocupacional em Pernambuco, até os anos 1990. Esta é a época em que há um incremento, no Brasil, o número de terapeutas ocupacionais titulados em nível de mestrado e doutorado, especialmente entre os docentes (EMMEL, 2001). No presente estudo, também encontramos a titulação de mestre apenas entre os entrevistados que são docentes.

Verificou-se discreta superioridade de atuação na área de Saúde Mental (44\%), em concordância com o estudo de Garcia (1997), que obteve em sua pesquisa

\footnotetext{
(1) Consulta aos documentos do Controle Acadêmico do Curso de Terapia Ocupacional / agosto 2003.
} 
mais de $60 \%$ de profissionais nesta área. Em nosso estudo, a existência de profissionais atuando em mais de uma área clínica é fruto de um mercado ainda em expansão, pelo fato de existir (até 2002) apenas um curso de Terapia Ocupacional no Recife, com reduzido número de vagas (30 vagas), colocando anualmente um pequeno número* desses profissionais no mercado local.

Em relação à modalidade de atendimento verificamos que a maioria dos terapeutas ocupacionais atende em ambulatório, em sessões grupal ou individual. As clínicas, consultórios e centros de reabilitação, com serviços prestados individualmente, foram por muitos anos o espaço mais freqüente de atuação do terapeuta ocupacional. O atendimento hospitalar e comunitário surgiu mais tardiamente, no final dos anos 1980, a partir da Reforma Sanitária e da reestruturação dos níveis de atenção à saúde, que englobou o terapeuta ocupacional também na assistência preventiva e coletiva (BRASIL, 1964, 2002; LEÃO et al., 2000).

Nos últimos tempos, a atenção grupal vem se expandindo, principalmente na saúde mental, pelo reconhecimento dos benefícios sociais e psíquicos que este atendimento proporciona (BENETTON, 1994). Segundo Garcia (1997), o atendimento grupal foi mais freqüente em seu estudo. O equilíbrio entre atendimento individual e grupal aqui referido pelos entrevistados pode ser explicado pela atuação em mais de uma área profissional.

Quanto à utilização de modelos teóricos em sua prática, $72 \%$ dos pesquisados responderam que usam tais modelos. O nosso achado é maior do que o de Garcia (1997), que encontrou 40\% dos terapeutas ocupacionais utilizando modelos como referencial teórico para a prática clínica. No entanto, esta é uma questão complexa em virtude das diferentes denominações atribuídas aos modelos, tanto pelos entrevistados como pela literatura. Se por um lado isto aponta para uma diversidade nas possibilidades, por outro confunde o entendimento e a comunicação entre os profissionais (HAGEDORN, 1999).

Outro aspecto em que não há consenso é quanto a classificação ou denominação das atividades terapêuticas. De acordo com a literatura, é através das atividades que o terapeuta ocupacional pode criar novas possibilidades e objetivos na intervenção, além de garantir várias formas de ação e expressão o que é mais importante do que a sua classificação (GILL, 1982; CASTRO et al., 2001). Por isto, adotamos a denominação referida pelos entrevistados, havendo o predomínio das atividades auto-expressivas, seguidas das artesanais, de vida diária e funcionais e atividades cognitivas ou pedagógicas. Garcia (1997) encontrou dificuldade em categorizar as atividades em seu trabalho, em virtude das diferentes denominações atribuídas pelos profissionais. Talvez a denominação similar que permitiu agrupar as respostas em nosso estudo deva-se à formação dos entrevistados em uma única universidade (apenas um entrevistado graduouse em outro Estado).

Em relação ao estudo da análise de atividades, $84 \%$ dos profissionais afirmaram que o assunto foi abordado no curso de graduação. A totalidade dos entrevistados acredita ser necessário o estudo da análise de atividade para a formação e $88 \%$ afirmaram ser muito importante para a prática do terapeuta ocupacional. Isto ratifica o pensamento de Crepeau (2002), para quem "a capacidade de analisar a atividade [...] está no centro da prática do terapeuta ocupacional” (p. 120).

Em se tratando da seleção de atividade para uso com a clientela atendida pelos pesquisados, a maioria $(52 \%)$ afirmou que escolhe a atividade a partir do interesse, necessidade ou decisão do(s) paciente(s). Encontramos essa referência na literatura que considera tais pontos no momento da escolha e indicação da atividade (SIMON, 1998; HAGEDORN, 1999; CREPEAU, 2002). O segundo lugar contou com $48 \%$ dos profissionais que relataram a escolha da atividade a partir da proposição dos objetivos terapêuticos, observando as habilidades, capacidades e aptidões dos pacientes, o que está em concordância com Hagedorn (1999) e Simon (1998), que afirmam que as atividades devem ser selecionadas buscando um objetivo definido para o paciente.

Um dado que merece destaque é que a amostra estudada compreende que a atividade tem grande relação com a pessoa a ser tratada, observando o contexto de vida, interesses, habilidades, etc., o que se assemelha aos dados da literatura que enfatizam o potencial terapêutico da atividade quando relacionada com as características do sujeito em tratamento. Isto pode ser explicado quando Castro et al. (2001) ressaltam que as atividades em Terapia Ocupacional não devem ser tidas como receitas, e sim, como uma conexão construída com cada paciente de forma singular.

Nosso estudo revelou que a maioria (84\%) dos pesquisados executa a análise de atividade em sua prática clínica, confirmando alguns trabalhos na literatura que incluem a análise de atividade como um procedimento do terapeuta ocupacional (FINLAY, 1988 apud BENETTON, 1994; MCGARRY, 1990 apud BENETTON,

\footnotetext{
* Consulta aos documentos do Controle Acadêmico do Curso de Terapia Ocupacional, agosto de 2003.
} 
1994). Por outro lado, $16 \%$ dos profissionais disseram que não fazem a análise de atividade. Acreditamos que isto pode relacionar-se ao fato de que a análise de atividade é um procedimento comum, incorporado à prática de maneira automática, diferenciando-se da forma que é realizada na formação acadêmica, não sendo por isso registrada (CREPEAU, 2002).

Ainda sobre esse assunto, nosso estudo obteve um percentual de $76 \%$ dos profissionais que não utilizam um roteiro específico para analisar as atividades. A literatura ressalta que é diferente a experiência da formação da aplicação da análise de atividade na prática clínica (CREPEAU, 2002).

No que se refere à definição da análise de atividade adotada pelos pesquisados, constatamos que a maioria a considerara como sendo a forma de se conhecer as características das atividades, ou como sendo a maneira de identificar seus benefícios e contra-indicações. Nesse sentido, verificou-se semelhança com a compreensão para a análise de atividades de autores como Hagedorn, (1999); Lamport et al. (1996); Punwar (1994) e Trombly (1989), que a conceituam como um procedimento que divide a atividade em suas partes componentes, buscando conhecer a sua natureza.

Por outro lado, para Crepeau (2002), esta compreensão situa-se na análise com ênfase na tarefa, o que é comum na etapa inicial da aprendizagem do estudante. Isto em nosso estudo estaria mantido entre os profissionais, embora também possa ser observada a presença da análise de atividades com ênfase no individuo (relação atividade-pessoa que a realizará), o que é mais evidenciando na vida profissional.

\section{CONCLUSÕES}

Para os terapeutas ocupacionais estudados, o conhecimento de análise de atividade é parte importante tanto da formação acadêmica como do exercício profissional, sendo que expressiva maioria realiza a análise de atividade em seu cotidiano sem, no entanto, utilizar um roteiro específico para este fim, conforme realizado durante a graduação.

A literatura aborda a análise da atividade, como parte de uma etapa da formação e do trabalho do terapeuta ocupacional, ora descrevendo-a como um processo de identificação das habilidades requeridas para o seu desempenho, ora como uma etapa mais avançada de apreensão da atividade em uso na prática clínica, envolvendo desde a discussão sobre os modelos de análise até a relação com o indivíduo. Constatamos existir relação entre a aprendizagem da análise de atividade e o seu uso na prática clínica conforme referem os entrevistados e o que está descrito na literatura especializada.

Por fim, com o estudo fica evidente que a análise de atividades além de ser um elemento presente na literatura, na legislação profissional e na formação do terapeuta ocupacional, está também, presente na prática cotidiana do terapeuta ocupacional preceptor de estágio da Universidade Federal de Pernambuco.

GUIMARÃES, D. S. L.; FALCÃO, I. V. Activity analysis and education of occupational therapists: a study with intern preceptors at the Federal University of Pernambuco, Brazil. Rev. Ter. Ocup. Univ. São Paulo, v. 15, n. 2, p. 63-70, maio/ago., 2004.

\begin{abstract}
ABSTRACT: The first undergraduate occupational therapy courses in Brazil were created in the late 1950s and early 1960s. In 1963, the minimum curriculum of occupational therapy courses came into being with approval from the National Education Council, and in 1982, this curriculum was modified, thus strengthening the specificity of the profession. In the early years of the 2000s, there occurred further change with the approval of the curricular directives, which indicated the necessity of studies on human activities and the analysis of such activities. The aim of the present work is to identify the course from professional education to the application of activity analysis in the practice of occupational therapist intern preceptor at the Federal University of Pernambuco, Brazil. This is a descriptive field study carried out with 28 curricular intern preceptors from the occupational therapy course of the Federal University of Pernambuco. A total of $96 \%$ of the individuals surveyed were women with between 10 and 19 years experience $(64 \%)$, predominantly in the areas of Mental Health and Neurology. The majority (88\%) of the interviewees stated having studied activity analysis during their undergraduate studies; all affirmed that the study of activity analysis was necessary for occupational therapists; and $84 \%$ carried out analysis in their day-today professional life. It is evident that activity analysis, besides constituting knowledge in the literature, professional legislation and the education of occupational therapists, is also present in the daily practices of professional intern preceptors at the Federal University of Pernambuco.
\end{abstract}

KEY WORDS: Occupational therapy/education. Occupational therapy/manpower. Curriculum/ trends. 


\section{REFERÊNCIAS}

BENETTON, M. J. A. Terapia ocupacional como instrumento nas ações de saúde mental. 1994. 190f. Tese (Doutorado) - Programa de Estudos Pós-Graduados em Saúde Mental da Faculdade de Ciências Médicas da UNICAMP, Campinas, 1994.

BRASIL. Ministério da Educação e Cultura. Currículo mínimo dos cursos de fisioterapia e terapia ocupacional. Parecer 388/63. Portaria ministerial 511/64, de 23 de julho de 1964.

BRASIL. Resolução N ${ }^{\circ} 04$ de 28 de fevereiro de 1983. Fixa os mínimos de conteúdo e duração dos cursos de fisioterapia e terapia ocupacional. Diário Oficial da República Federativa do Brasil, Poder Executivo, Brasília, DF, 7 mar.1983.

BRASIL. Ministério da Educação e do Desporto. Conselho Nacional de Educação. Orientação para as diretrizes curriculares dos cursos de graduação. Parecer nº 776 de 1997. 2002. Disponível em: http://www.pol.org.br. Acesso em: 25 ago.

BRASIL. Resolução ${ }^{\circ} 06$ de 19 de fevereiro de 2002. Institui diretrizes curriculares nacionais do curso de graduação em terapia ocupacional. Diário Oficial da República Federativa do Brasil, Poder Executivo, Brasília, DF, 4 mar. 2002. seção 1, p. 12 .

CASTRO, E. D; LIMA, E. M. F. A.; BRUNELLO, M. I. B. Atividades humanas e terapia ocupacional. In: DE CARLO, M. M. R. P.; BARTAlOTTI, C. C. (Org.). Terapia ocupacional no Brasil. Fundamentos e perspectivas. São Paulo: Plexus, 2001. p. 41-59.

CREPEAU, E. B. Análise de atividades: uma forma de refletir sobre desempenho ocupacional. In: NEISTADT, M. E.; CREPEAU, E. B. Terapia ocupacional. Rio de Janeiro: Guanabara Koogan, 2002. Cap.12, p. 121-133.

DE CARLO, M. M. R. P; BARTALOTTI, C. C. (Org.). Terapia ocupacional no Brasil. Fundamentos e perspectivas. São Paulo: Plexus, 2001. p. 19-40: Caminhos da terapia ocupacional.

EMMEL, M. L. G. A questão da formação. 2001. Disponível em: http://www.togyn.tripod.com.br. Acesso em: 02 set. 2002.

EMMEL, M. L. G; LANCMAN, S. Quem são nossos mestres e doutores? O avanço da capacitação docente em terapia ocupacional no Brasil. Cad. Ter. Ocup. UFSCar, São Carlos, v. 7, n. 1, p. 29-38, jan-jun. 1998.

FERRIGNO, I. S. V. Terapia ocupacional: considerações sobre o contexto profissional. Rev. Ter. Ocup. Univ. São Paulo. São Paulo, v. 2, n. 1, fev., 1991.

FINGER, J. A. O. Aspectos gerais. In: FINGER, J. A. O
Terapia ocupacional. São Paulo: Sarvier, 1986. p.1-13.

GARCIA, M. I. S. Formação do terapeuta ocupacional. In: Congresso Brasileiro de Terapia Ocupacional, 5., SIMPÓSIO LATINO-AMERICANO DE TERAPIA OCUPACIONAL, 4., Belo Horizonte, 1997. Anais. Belo Horizonte, 1997. p. 120-126.

GILL, N. B. Uma visão global do atendimento de terapia ocupacional. J. Bras. Reabil., Niterói, v. 3, n. 9, p. 14-17, jan-mar. 1982.

HAGEDORN, R. Habilidades e processos essenciais. In: HAGEDORN, R. Fundamentos da prática em terapia ocupacional. São Paulo: Dynamis editorial, 1999. Cap. 3, p. $29-48$.

HAGEDORN, R. As bases filosóficas e teóricas da prática. In: HAGEDORN, R. Fundamentos da prática em terapia ocupacional. São Paulo: Dynamis editorial, 1999. Cap. 4, p. $49-68$.

LAMPORT, N. K; COFFEY, M. S; HERSCH, G. I. Examining the process. In: LAMPORT, N. K; COFFEY, M. S; HERSCH, G. I. Activity analysis and application: building blocks of treatment. USA: Slack, 1993. Cap. 1, p. 1-11.

LEÃO, A. et al. A terapia ocupacional e as políticas públicas de saúde da cidade de São Paulo. Cad. Ter. Ocup. UFSCar, São Carlos, v. 8, n. 1, p. 48-56, jan-jun. 2000.

PALHARES, M. S. Estudo do currículo de terapia ocupacional. Cad. Ter. Ocup. UFSCar, São Carlos, v. 2, n. 2, jul-dez. 1991.

PESSOA, A. I. S. V. Terapia ocupacional: entraves acadêmicos na projeção do profissional. [Projeto de pesquisa] - Departamento de Reabilitação, Universidade Federal de Pernambuco, Recife, 1992.

PUNWAR, A. J. Use of activity as therapeutic tool. In: PUNWAR, A. J. Occupation therapy - principles and pratice. 2a ed. Baltimore: Williams e Wilkins, 1994. Cap. 4, p. 35-46.

ROSA, S. D; EMMEL, M. L. G. Reflexões sobre os diferentes papéis assumidos pelo terapeuta ocupacional enquanto professor universitário. Cad. Ter. Ocup. UFSCar, São Carlos, v. 9, n. 1, jan-jun. 2001.

SIMON, C. J. Uso de la actividad y análisis de actividad. In: HOPKINS, H. L.; SMITH, H. D. Terapia ocupacional. 8. ed. Madrid: Panamericana, 1998. Cap 9, sección 3, p. 281-292.

SOARES, L. B. T. Terapia ocupacional: do reducionismo a uma práxis unitária. In: SOARES, L. B. T. Terapia ocupacional: lógica do capital ou do trabalho? São Paulo: Hucitec, 1991. p. 139-204. 
GUIMARÃES, D. et al.. Análise de atividades do TO. Rev. Ter. Ocup. Univ. São Paulo, v. 15, n. 2, p. 63-70, maio/ago., 2004.

SOARES, L. B. T. Diretrizes curriculares - avaliação. In: ENCONTRO NACIONAL DE DOCENTES DE TERAPIA OCUPACIONAL, 7., Salvador, BA, 2000. Relatório final. Salvador, BA, 2000.

SOUZA, C. T. C. Perfil do terapeuta ocupacional brasileiro. In: Congresso Brasileiro de Terapia Ocupacional, 5., SIMPÓSIO LATINO-AMERICANO DE TERAPIA OCUPACIONAL, 4., 1997. Anais. Belo Horizonte, 1997. p. 5-16.

Recebido para publicação: 28/04/04

Aceito para publicação: 01/06/04
TROMBLY, C. A. Seleção e análise de atividades. In: TROMBLY, C. A. Terapia ocupacional para a disfunção física. 2. ed. São Paulo: Santos, 1989. p. 243-248.

VITTA, F. C. F. Formação do terapeuta ocupacional que atua com crianças com atraso no desenvolvimento neuropsicomotor. In: VITTA, F. C. F. Uma identidade em construção: o terapeuta ocupacional e a criança retardo no desenvolvimento neuropsicomotor. Bauru, SP: Edusc, 1998. Cap. IV, p. 57-77. 